\title{
Soft-X-Ray Harmonic Comb from Relativistic Electron Spikes
}

\author{
A. S. Pirozhkov, ${ }^{1}$ M. Kando, ${ }^{1}$ T. Zh. Esirkepov, ${ }^{1}$ P. Gallegos,,${ }^{2,3}$ H. Ahmed, ${ }^{4}$ E. N. Ragozin, ${ }^{5}$ A. Ya. Faenov, ${ }^{1,6}$ \\ T. A. Pikuz, ${ }^{1,6}$ T. Kawachi, ${ }^{1}$ A. Sagisaka, ${ }^{1}$ J. K. Koga, ${ }^{1}$ M. Coury, ${ }^{3}$ J. Green, ${ }^{2}$ P. Foster, ${ }^{2}$ C. Brenner, ${ }^{2,3}$ B. Dromey, ${ }^{4}$ \\ D. R. Symes,${ }^{2}$ M. Mori,${ }^{1}$ K. Kawase, ${ }^{1}$ T. Kameshima, ${ }^{1}$ Y. Fukuda, ${ }^{1}$ L. Chen, ${ }^{1}$ I. Daito, ${ }^{1}$ K. Ogura, ${ }^{1}$ Y. Hayashi, ${ }^{1}$ H. Kotaki, ${ }^{1}$ \\ H. Kiriyama, ${ }^{1}$ H. Okada, ${ }^{1}$ N. Nishimori, ${ }^{7}$ T. Imazono, ${ }^{1}$ K. Kondo, ${ }^{1}$ T. Kimura, ${ }^{1}$ T. Tajima, ${ }^{1}$ H. Daido, ${ }^{1}$ P. Rajeev, ${ }^{2}$ \\ P. McKenna, ${ }^{3}$ M. Borghesi, ${ }^{4}$ D. Neely, ${ }^{2,3}$ Y. Kato, ${ }^{1}$ and S. V. Bulanov ${ }^{1,8}$ \\ ${ }^{1}$ Advanced Beam Technology Division, JAEA, 8-1-7 Umemidai, Kizugawa, Kyoto 619-0215, Japan \\ ${ }^{2}$ Central Laser Facility, Rutherford Appleton Laboratory, STFC, Chilton, Didcot, Oxon OX11 OQX, United Kingdom \\ ${ }^{3}$ Department of Physics, University of Strathclyde, SUPA, Glasgow G4 ONG, United Kingdom \\ ${ }^{4}$ Centre for Plasma Physics, The Queen's University of Belfast, Belfast BT7 1NN, United Kingdom \\ ${ }^{5}$ P. N. Lebedev Physical Institute, RAS, Leninsky prospekt 53, Moscow 119991, Russia \\ ${ }^{6}$ Joint Institute of High Temperatures, RAS, Izhorskaja Street 13/19, Moscow 127412, Russia \\ ${ }^{7}$ Laser Application Technology Division, JAEA, 2-4 Shirakata-Shirane, Tokai, Ibaraki 319-1195, Japan \\ ${ }^{8}$ A. M. Prokhorov Institute of General Physics, RAS, Vavilov Street 38, Moscow 119991, Russia
}

(Received 13 March 2011; published 30 March 2012)

\begin{abstract}
We demonstrate a new high-order harmonic generation mechanism reaching the "water window" spectral region in experiments with multiterawatt femtosecond lasers irradiating gas jets. A few hundred harmonic orders are resolved, giving $\mu \mathrm{J} / \mathrm{sr}$ pulses. Harmonics are collectively emitted by an oscillating electron spike formed at the joint of the boundaries of a cavity and bow wave created by a relativistically self-focusing laser in underdense plasma. The spike sharpness and stability are explained by catastrophe theory. The mechanism is corroborated by particle-in-cell simulations.
\end{abstract}

High-order harmonic generation, one of the most fundamental effects of nonlinear wave physics, originates from many nonlinearities, e.g., relativistic $[1,2]$ and ionizing matter effects [3]. Harmonics reaching the x-ray spectral region enable nanometer and attosecond resolution in biology, medicine, physics, and their applications. Several compact laser-based $\mathrm{x}$-ray sources have been implemented, including plasma-based x-ray lasers [4], atomic harmonics in gases [3], nonlinear Thomson scattering [5] from plasma electrons [6,7] and electron beams [8], betatron radiation [9], relativistic flying mirrors [10], and harmonics from solid targets [1,2,11]. Many of them [1,5-11] come from relativistic laser-matter interactions, determined by the dimensionless laser pulse amplitude $a_{0}=$ $e E_{0} / m_{e} c \omega_{0}>1$ related to the laser peak irradiance by $I_{0 L}=I_{\text {rel }} a_{0}^{2}\left(\mu \mathrm{m} / \lambda_{0}\right)^{2}$ for linear and $I_{0 C}=2 I_{0 L}$ for circular polarization. Here, $e$ and $m_{e}$ are the electron charge and mass; $c$ is the speed of light in a vacuum; $\omega_{0}, \lambda_{0}$, and $E_{0}$ are the laser angular frequency, wavelength, and peak electric field, respectively; and $I_{\text {rel }}=1.37 \times 10^{18} \mathrm{~W} / \mathrm{cm}^{2}$ [2].

In this Letter, we experimentally demonstrate bright extreme ultraviolet and soft x-ray harmonics resolved up to a few hundred orders from gas jets irradiated by multiterawatt femtosecond lasers. We propose a new mechanism of high-order harmonics generation.

We have performed two experimental campaigns using J-KAREN [12] and Astra Gemini [13] lasers. The laser pulse power $P_{0}$, duration $\tau$, wavelength $\lambda_{0}$, off-axis parabolic mirror $f$ number, and irradiance in a vacuum are
$9 \mathrm{TW}, 27 \mathrm{fs}, 820 \mathrm{~nm}, f / 9$, and $4 \times 10^{18} \mathrm{~W} / \mathrm{cm}^{2}$ in the first campaign and $120 \mathrm{TW}, 54 \mathrm{fs}, 804 \mathrm{~nm}, f / 20$, and $4 \times 10^{18} \mathrm{~W} / \mathrm{cm}^{2}$ in the second campaign, respectively. Focusing laser pulses onto a supersonic helium gas jetsee Fig. 1 -we record harmonics in the $80-360 \mathrm{eV}$ spectral region in the forward (laser propagation) direction-see Figs. 2 and 3. We use flat-field spectrographs [14], comprising a gold-coated grazing-incidence collecting mirror, spherical varied-line-space grating, and back-illuminated charge coupling device (CCD), shielded by optical blocking filters-two $0.16 \mu \mathrm{m}$ multilayer Mo/C, two $0.2 \mu \mathrm{m}$ $\mathrm{Pd}$, or one $0.2 \mu \mathrm{m} \mathrm{Ag}$ on a $0.1 \mu \mathrm{m} \mathrm{CH}$ substrate. The acceptance angle is $3 \times 10^{-5} \mathrm{sr}$ in the first and $3 \times 10^{-6} \mathrm{sr}$ in the second campaign. In the latter case, the spectrograph has two channels for observation at $0^{\circ}$ (forward direction) and $0.53^{\circ}$ in the laser (linear) polarization plane. The wavelength calibration is performed in place, using spectra of Ar and Ne plasmas. The harmonics energy and photon numbers are conservatively estimated using idealized spectrograph throughputs, i.e., the products of the calculated [15] collecting mirror reflectivities and

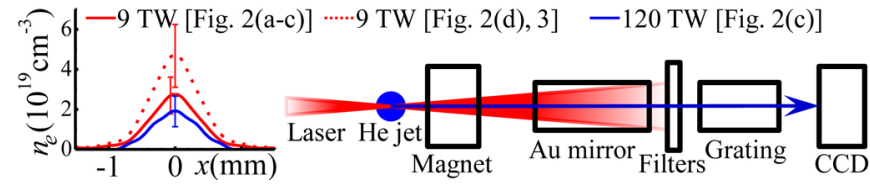

FIG. 1 (color online). The experiment scheme (right) and $\mathrm{He}$ plasma density profile (left) for the shots in Figs. 2 and 3. 


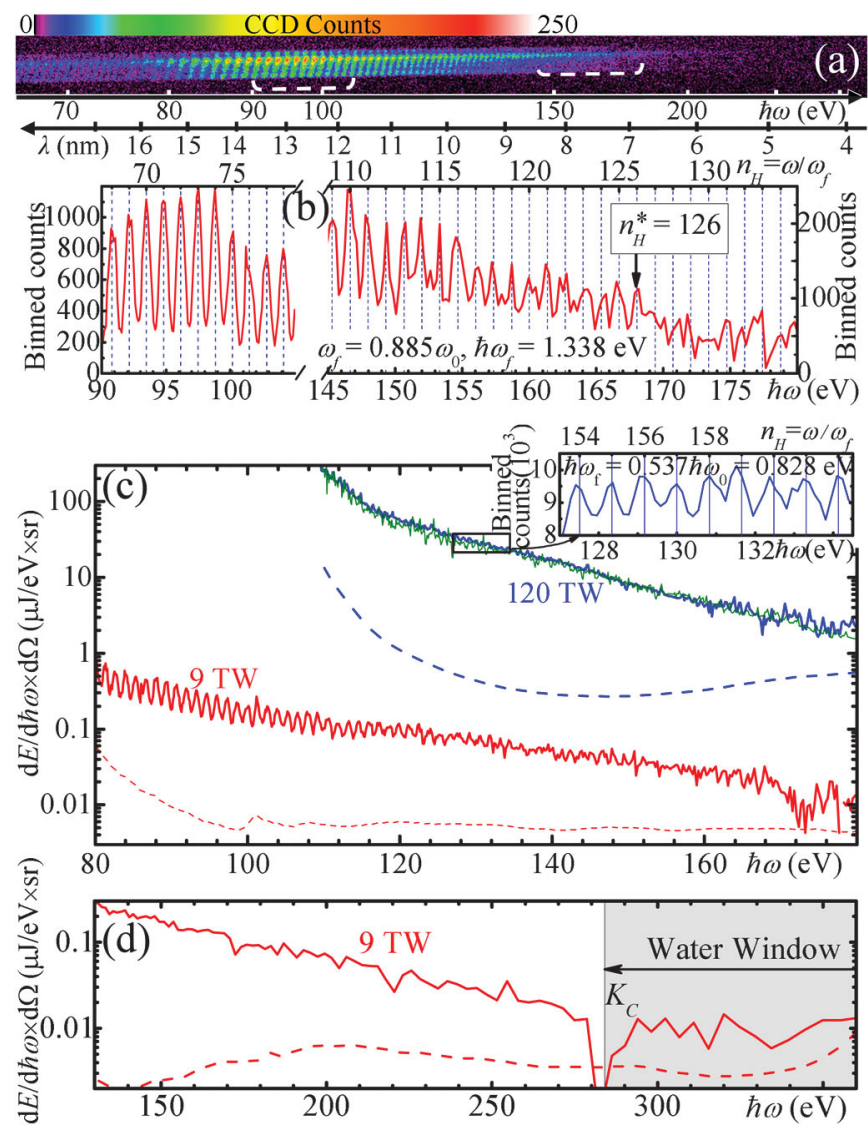

FIG. 2 (color). Typical single-shot comblike spectra. (a) Raw data for $P_{0}=9 \mathrm{TW}, n_{e}=2.7 \times 10^{19} \mathrm{~cm}^{-3}$. (b) Curves for two regions marked in (a) by dashed brackets. Dotted vertical lines are for harmonics of the base frequency $\omega_{f}=0.885 \omega_{0}$. The highest resolved order is $n_{H}^{*}=126$. (c) Spectra for the shot shown in (a) (red) and for $P_{0}=120 \mathrm{TW}, n_{e}=1.9 \times 10^{19} \mathrm{~cm}^{-3}$ obtained from the 2 nd (blue) and 3rd (green) diffraction orders. Inset: harmonics in the 2nd diffraction order; similarly for the 3rd order. (d) The spectral region embracing the "water window" for $P_{0}=9 \mathrm{TW}, n_{e}=4.7 \times 10^{19} \mathrm{~cm}^{-3}$. The drop at the carbon $K$ absorption edge, $K_{C}$, is due to hydrocarbon contamination. Dashed curves in (c),(d) are for background due to the CCD dark current, readout noise, and scattered photons.

filter transmissions, measured diffraction grating efficiencies, and manufacturer-provided CCD efficiencies.

In both campaigns, the laser power significantly exceeds the relativistic self-focusing threshold, $P_{\mathrm{sf}} \approx 17 \mathrm{GW} \times\left(n_{\mathrm{cr}} / n_{e}\right), \quad$ where $n_{\mathrm{cr}}=m \omega_{0}^{2} / 4 \pi e^{2} \approx$ $1.1 \times 10^{21} \mathrm{~cm}^{-3}\left(\mu \mathrm{m} / \lambda_{0}\right)^{2}$ is critical density. Thus, both lasers' pulses self-focus to tighter spots, reaching higher irradiance determined by the plasma density and laser power.

The comblike spectra comprising even and odd harmonic orders of similar intensity and shape are generated by both linearly and circularly polarized pulses in a broad range of plasma electron densities from $n_{e} \simeq 1.7 \times 10^{19}$ to $7 \times 10^{19} \mathrm{~cm}^{-3}$. The harmonic base frequency $\omega_{f}$ is downshifted from the laser frequency $\omega_{0}$ - see

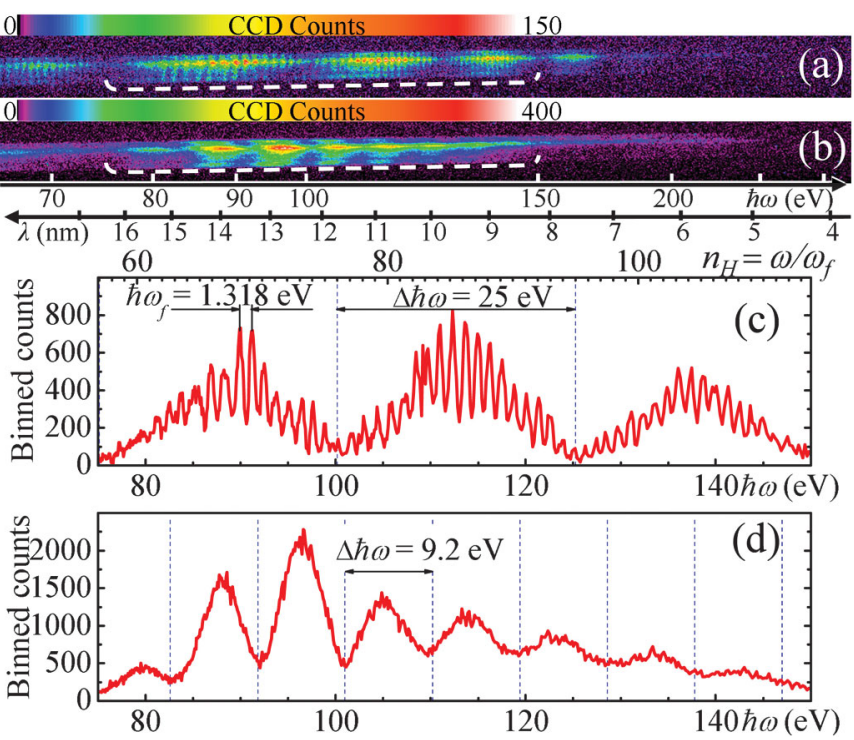

FIG. 3 (color). Modulated spectra with (a) resolved and (b) nearly unresolved harmonic structure for $P_{0}=9 \mathrm{TW}, n_{e}=$ $4.7 \times 10^{19} \mathrm{~cm}^{-3}$. Curves in (c),(d) show spectra denoted by dashed brackets in (a),(b), respectively.

Figs. 2(b) and 2(c) - in correlation with the transmitted laser spectra.

The data obtained with different lasers demonstrate the effect's reproducibility and robustness and the photon yield scalability with the laser power-see Fig. 2(c). For the $120 \mathrm{TW}$ laser, the photon number and energy in unit solid angle within one harmonic at $120 \mathrm{eV}$ are $2 \times 10^{12}$ photons $/ \mathrm{sr}$ and $40 \mu \mathrm{J} / \mathrm{sr}$, respectively. These amount to $4 \times 10^{9}$ photons and $90 \mathrm{~nJ}$, assuming the $1.5^{\circ}$ angular radius inferred from our particle-in-cell (PIC) simulations, which agrees with similar spectral intensities observed at 0 and $0.53^{\circ}$ channels. Figure 2(d) shows the spectrum up to the spectrograph throughput cutoff of $360 \mathrm{eV}$. The emission reaches the "water window" region (284-543 eV), required for high-contrast femtosecond bioimaging, where the $9 \mathrm{TW}$ laser produces $\left(1.5_{-0.4}^{+0.5}\right) \times 10^{10}$ photons $/ \mathrm{sr}$ and $0.8_{-0.2}^{+0.3} \mu \mathrm{J} / \mathrm{sr}$, corresponding to $3 \times 10^{7}$ photons and $1.7 \mathrm{~nJ}$ for the same $1.5^{\circ}$ radius. The uncertainties are due to the $10 \%$ filter thickness tolerance and CCD noise.

A large number of resolved harmonics, e.g., $n_{H}^{*} \simeq 126$ in Figs. 2(a) and 2(b) and $\gtrsim 160$ in Fig. 2(c) (inset), strictly bounds the laser frequency change during the harmonic emission process, $\delta \omega / \omega \leq 1 /\left(2 n_{H}^{*}\right)$; otherwise, the orders $n_{H}^{*}$ and $n_{H}^{*}+1$ overlap. The laser frequency decreases due to an adiabatic depletion of the laser pulse losing energy on plasma waves, while the number of photons is conserved [16-18]. For slow depletion, the frequency downshift rate equals the energy depletion rate, $\delta \omega / \omega=-\delta x / L_{\mathrm{dep}}$ [16-18]. For Figs. 2(a) and 2(b), the depletion length is $L_{\text {dep }} \approx 2.7 \mathrm{~mm}$, estimated from the observed $70 \%$ energy transmission through the $0.9 \mathrm{~mm}$ plasma. Estimate [18] 
gives a similar value, $L_{\mathrm{dep}} \approx 8.7\left(n_{\mathrm{cr}} / n_{e}\right)^{3 / 2} \lambda \approx 3.4 \mathrm{~mm}$. The condition $\delta \omega / \omega<0.4 \%$ (or, alternatively, the phase error of $<25 \mathrm{mrad}$ ) gives the harmonic emission length of $\lesssim 12 \mu \mathrm{m}$. We note that the emission length can be much longer than the longitudinal size of the moving source.

In many shots with linear polarization $(40 \%$ with the 9 TW laser), the harmonic spectrum exhibits deep equidistant modulations-see Fig. 3. They are visible with discernible high orders and even with nearly unresolved individual harmonics in some shots-see Figs. 3(b) and 3(d) - where blurring can be caused by a greater downshift of the laser frequency during a longer emission of harmonics, consistently exhibiting a few times greater photon number. Since in some cases the modulation depth is $\sim 100 \%$, we conclude that the modulations result from interference between two almost identical strongly localized coherent sources separated in time and/or space. In shots with circularly polarized pulses ( 90 shots with the 120 TW laser), large-scale spectral modulations are not observed.

The unique properties of the observed harmonics mismatch previously suggested mechanisms of high-order harmonics generation. Atomic harmonics are excluded because both linearly and circularly polarized laser pulses produce both even and odd harmonic orders with a weak sensitivity to gas pressure. Betatron radiation is not relevant because the base frequency of its harmonics is determined by the plasma frequency and electron energy, not the laser frequency. Nonlinear Thomson scattering cannot provide the observed photon numbers even under the most favorable assumptions. For the 9 TW shot-see Figs. 2(a)-2(c) - the numerical calculation of singleelectron radiation spectra [7,19] gives at $100 \mathrm{eV}$ at most $2 \times 10^{-10} \mathrm{~nJ} / \mathrm{eV} \mathrm{sr}$, assuming the self-focused laser amplitude of $a_{0}=7$, pulse duration of $30 \mathrm{fs}$, and the observation angle of $15^{\circ}$ close to optimum [7], where the high-frequency nonlinear Thomson scattering component is most intense. The number of electrons encountered by the laser pulse is $N_{e}=\pi d_{\mathrm{sf}}^{2} \Delta x n_{e} / 4 \approx 6 \times 10^{9}$, where $n_{e}=2.7 \times 10^{19} \mathrm{~cm}^{-3}, d_{\mathrm{sf}} \approx 5 \mu \mathrm{m}$ is the self-focusing channel diameter [20], and $\Delta x \approx 12 \mu \mathrm{m}$ is the harmonics emission length (see above). This gives at most $1 \mathrm{~nJ} / \mathrm{eV} \mathrm{sr}$, 200 times smaller than experimentally observed. For other spectra, shown in Figs. 2(c) and 2(d), an even smaller fraction corresponds to nonlinear Thomson scattering.

We performed two- (2D) and three-dimensional (3D) PIC simulations of harmonics generation using the code REMP [21]. As seen in simulations [22], the laser pulse undergoes self-focusing $[2,17]$ and pushes electrons out, evacuating a cavity in electron density $[17,23]$ and generating a bow wave [24]. Figure 4(a) represents the case when a linearly polarized (along the $y$ axis) laser pulse, self-focused to the amplitude of $a_{0}=6.6$ and FWHM waist of $10 \lambda_{0}$, reaches a point where $n_{e}=1.8 \times 10^{18} \mathrm{~cm}^{-3}$. The simulation window size is

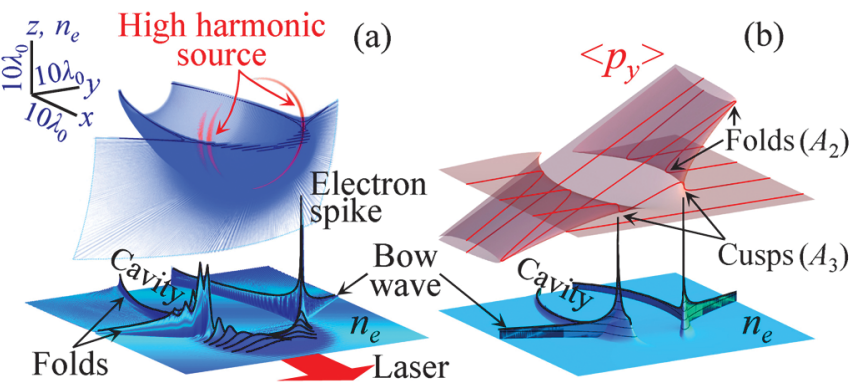

FIG. 4 (color). (a) 3D PIC simulation. Electron density $n_{e}$ (top, upper half removed) and its cross section at $z=0$ (bottom). The electromagnetic energy density for $\omega \geq 4 \omega_{0}$ is represented by red arcs. (b) Catastrophe theory model. Singularities in the electron density are created by foldings of the electron phase space $\left(x, y,\left\langle p_{y}\right\rangle\right)$, where $p_{y}$ is averaged over the laser period.

$125 \lambda_{0} \times 124 \lambda_{0} \times 124 \lambda_{0}$, with the resolution of $d x=\lambda_{0} / 32$ and $d y=d z=\lambda_{0} / 8$; the number of quasiparticles is $2.3 \times 10^{10}$; and ions are immobile. The electromagnetic energy density of a high-frequency $\left(\omega \geq 4 \omega_{0}\right)$ field reveals the source of high-order harmonics [see Fig. 4(a) (red arcs)]: they are emitted by the oscillating electron density spikes formed at the joining of the bow wave and cavity boundaries. While emission is also seen from the cavity walls [25], at higher frequencies, much stronger radiation originates from the electron spikes.

The high-order harmonics emitted by the electron spikes are seen in 2D PIC simulation, performed in the $87 \lambda_{0} \times 72 \lambda_{0}$ window with $d x=\lambda_{0} / 1024, d y=\lambda_{0} / 112$, and $6 \times 10^{8}$ quasiparticles. In Fig. 5, the laser pulse acquiring the amplitude of $a_{0}=10$, the duration of $16 \lambda_{0}$, and the waist of $10 \lambda_{0}$ meets plasma with $n_{e}=$ $1.7 \times 10^{19} \mathrm{~cm}^{-3}$. Starting from the 7th order, harmonics are well discernible up to the 128th order, as allowed by the simulation resolution. The emission is slightly off-axis with the angle decreasing with increasing harmonic order.

A strong localization of electron spikes, their robustness to oscillations imposed by the laser and, ultimately, their superior contribution to high-order harmonics emission, is explained by catastrophe theory [26]. The laser pulse creates a multistream electron flow [27,28], stretching and folding an initially flat surface formed by electrons in their phase space-see Fig. 4(b) and Ref. [22]. The surface projection onto the $(x, y)$ plane gives the electron density where outer and inner folds are mapped into singular curves outlining the bow wave and cavity boundaries, respectively. Catastrophe theory here establishes universal structurally stable singularities, insensitive to perturbations. The bow wave and cavity boundaries produce the "fold"-type singularity $\left(A_{2}\right.$, according to Arnold's classification [26]), where the density grows as $(\Delta y)^{-1 / 2}$ with decreasing distance to the boundary, $\Delta y$. At the joint of the two folds, the density grows as $(\Delta y)^{-2 / 3}$, forming a higher-order singularity - the "cusp" $\left(A_{3}\right)$ - see Figs. 5(c) and 5(d) and Ref. [22]. Stronger singularities exist [28]; 


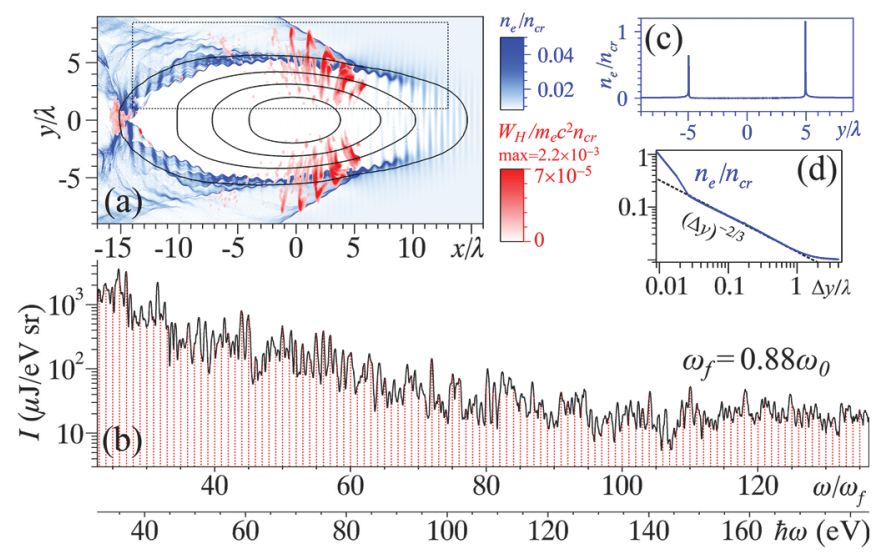

FIG. 5 (color). 2D PIC simulation. (a) The electron density (blue), laser envelope (curves for $a=1,4,7,10$ ), and electromagnetic energy density, $W_{H}$, for frequencies from 60 to $100 \omega_{0}$ (red). (b) The upper spike emission spectrum for the dashed rectangle in (a). (c) The electron density profile 10 laser cycles earlier than (a) and (d) the right spike structure.

however, they are not stable against perturbations. Located in a ring surrounding the cavity head, the cusp is seen in simulations as an electron spike. For linear polarization, the harmonic emitting ring breaks up into two segmentssee Fig. 4(a). The spike oscillations imposed by the laser generate high-order harmonics. The cusp singularity ensures a tight concentration of electric charge, making the emission coherent, i.e., the intensity is proportional to the particle number squared $N_{e}^{2}$, similarly to the coherent nonlinear Thomson scattering. However, the cusp consists of different particles at every moment of time, in contrast to a synchronous motion of the same particles. We note that, for constructive interference, it is sufficient that the source size is smaller than the emitted wavelength in the direction of observation only. The estimated number of electrons within the singularity ring, $N_{e} \sim 10^{6}$, provides a signal level close to the experiment.

In classical electrodynamics [19], an oscillating electron emits harmonics up to critical order $n_{H c}$, proportional to the cube of the particle energy $\mathcal{E}_{e} \approx a_{0} m c^{2}$,

$$
n_{H c}=\omega_{c} / \omega_{f} \sim a_{0}^{3} ;
$$

then, the spectrum exponentially vanishes, as seen in our experiments. This gives clues about the harmonics generation scaling. For the laser dimensionless amplitude in the stationary self-focusing [20], $a_{0, \mathrm{sf}}=\left(8 \pi P_{0} n_{e} / P_{c} n_{\mathrm{cr}}\right)^{1 / 3}$, we obtain the critical harmonic order of $n_{H c} \sim$ $P_{0} n_{e} / P_{c} n_{\mathrm{cr}}$, where $P_{c}=2 m_{e}^{2} c^{5} / e^{2} \approx 17 \mathrm{GW}$. The total energy $\mathcal{E}_{s}$ emitted by the electron spike [19] is proportional to $N_{e}^{2}$ and the harmonic emission time $\tau_{H}$ :

$$
\mathcal{E}_{s} \approx e^{2} N_{e}^{2} a_{0}^{4} \gamma \omega_{0}^{2} \tau_{H} / 8 c \propto N_{e}^{2} P_{0}^{4 / 3} n_{e}^{5 / 6} \omega_{0}^{1 / 3} \tau_{H} .
$$

Here, $\gamma \approx\left(n_{c r} / n_{e}\right)^{1 / 2}$ is the Lorentz factor associated with the spike velocity, which is close to the laser pulse group velocity in plasma. In the $120 \mathrm{TW}$ shot—see Fig. 2(c)—the energy emitted into a harmonic near $120 \mathrm{eV}$ is $40 \mu \mathrm{J} / \mathrm{sr}$. In the 2D PIC simulation with the parameters close to this shot (Fig. 5), the energy of the 100th harmonic is $30 \mu \mathrm{J} / \mathrm{sr}$. An estimate based on Eq. (2) gives $100 \mu \mathrm{J} / \mathrm{sr}$ within the 100th harmonic, thus providing a simple analytical estimate for the expected harmonic energy.

With the detector at $1.4 \mathrm{~m}$ and the source size of $10 \mu \mathrm{m}$ estimated from simulations, the spatial coherence width is $1 \mathrm{~mm}$, which is large enough for many phase contrast imaging applications in a compact setup [29].

In conclusion, in irradiating gas jets with multiterawatt lasers, we observe comblike harmonic spectra reaching the "water window" region. We propose the harmonic generation mechanism based on self-focusing, nonlinear wave formation with electron density spikes, and collective radiation by a compact electric charge. Catastrophe theory explains the electron spike sharpness and stability. The maximum harmonic order scaling with the laser intensity will allow reaching the $\mathrm{keV}$ range. Our results open the way to a compact coherent $\mathrm{x}$-ray source built on a university laboratory scale repetitive laser and an accessible, replenishable, and debris-free gas jet target. This will impact many areas requiring a bright $\mathrm{x}$-ray or extreme ultraviolet source for pumping, probing, imaging, or attosecond science.

We acknowledge the financial support from MEXT (Kakenhi 20244065, 21604008, 21740302, and 23740413), the JAEA President Grant, and the STFC facility access fund.

[1] U. Teubner and P. Gibbon, Rev. Mod. Phys. 81, 445 (2009).

[2] G. A. Mourou et al., Rev. Mod. Phys. 78, 309 (2006).

[3] A. McPherson et al., J. Opt. Soc. Am. B 4, 595 (1987); P. B. Corkum, Phys. Rev. Lett. 71, 1994 (1993); F. Krausz and M. Ivanov, Rev. Mod. Phys. 81, 163 (2009).

[4] D. L. Matthews et al., Phys. Rev. Lett. 54, 110 (1985); S. Suckewer et al., ibid. 55, 1753 (1985); H. Daido, Rep. Prog. Phys. 65, 1513 (2002).

[5] E. Esarey, S. K. Ride, and P. Sprangle, Phys. Rev. E 48, 3003 (1993).

[6] S. Y. Chen et al., Nature (London) 396, 653 (1998); Phys. Rev. Lett. 84, 5528 (2000); K. Ta Phuoc et al., ibid. 91, 195001 (2003); S. Banerjee et al., J. Opt. Soc. Am. B 20, 182 (2003).

[7] K. Lee et al., Phys. Rev. E 67, 026502 (2003).

[8] R.W. Schoenlein et al., Science 274, 236 (1996); M. Babzien et al., Phys. Rev. Lett. 96, 054802 (2006).

[9] F. Albert et al., Plasma Phys. Controlled Fusion 50, 124008 (2008); S. Kneip et al., Nature Phys. 6, 980 (2010).

[10] S. V. Bulanov, T. Esirkepov, and T. Tajima, Phys. Rev. Lett. 91, 085001 (2003); M. Kando et al., ibid. 99, 135001 (2007); 103, 235003 (2009); A. S. Pirozhkov et al., Phys. Plasmas 14, 123106 (2007). 
[11] S. V. Bulanov et al., Phys. Plasmas 1, 745 (1994); B. Dromey et al., Nature Phys. 2, 456 (2006); C. Thaury et al., ibid. 3, 424 (2007); Y. Nomura et al., ibid. 5, 124 (2009).

[12] H. Kiriyama et al., Opt. Lett. 33, 645 (2008).

[13] C. J. Hooker et al., J. Phys. IV 133, 673 (2006).

[14] I. W. Choi et al., Appl. Opt. 36, 1457 (1997); D. Neely et al., in Superstrong Fields in Plasmas, edited by M. Lontano, G. Mourou, F. Pegoraro, and E. Sindoni, AIP Conf. Proc. No. 426 (AIP, New York, 1998), p. 479.

[15] B.L. Henke et al., At. Data Nucl. Data Tables 54, 181 (1993); http://henke.lbl.gov/optical_constants/.

[16] S. V. Bulanov et al., Phys. Fluids B 4, 1935 (1992).

[17] E. Esarey et al., Rev. Mod. Phys. 81, 1229 (2009).

[18] B. A. Shadwick et al., Phys. Plasmas 16, 056704 (2009).

[19] J.D. Jackson, Classical Electrodynamics (Wiley, New York, 1998), 3rd ed.

[20] S. S. Bulanov et al., Phys. Plasmas 17, 043105 (2010).
[21] T.Z. Esirkepov, Comput. Phys. Commun. 135, 144 (2001).

[22] See Supplemental Material at http://link.aps.org/ supplemental/10.1103/PhysRevLett.108.135004 for Supplemental Movies S1, S2, and S3.

[23] A. Pukhov and J. Meyer-ter-Vehn, Appl. Phys. B 74, 355 (2002).

[24] T.Z. Esirkepov, Y. Kato, and S. V. Bulanov, Phys. Rev. Lett. 101, 265001 (2008).

[25] D. F. Gordon et al., Phys. Rev. Lett. 101, 045004 (2008).

[26] T. Poston and I. Stewart, Catastrophe Theory and Its Applications (Dover, New York, 1996).

[27] S. V. Bulanov et al., Phys. Rev. Lett. 78, 4205 (1997).

[28] A. V. Panchenko et al., Phys. Rev. E 78, 056402 (2008).

[29] S. W. Wilkins et al., Nature (London) 384, 335 (1996); H. N. Chapman and K. A. Nugent, Nature Photon. 4, 833 (2010); B. Abbey et al., ibid. 5, 420 (2011). 